Dieses Dokument ist eine Zweitveröffentlichung (Postprint) /

This is a self-archiving document (accepted version):

M. Hoffmann, S. Slesazeck, T. Mikolajick

Domain Formation in Ferroelectric Negative Capacitance Devices

Erstveröffentlichung in / First published in:

76th Device Research Conference (DRC). Santa Barbara, 2018. IEEE. ISBN 978-1-5386-3028-0

DOI: https://doi.org/10.1109/DRC.2018.8442139

Diese Version ist verfügbar / This version is available on:

https://nbn-resolving.org/urn:nbn:de:bsz:14-qucosa2-768342 


\title{
Domain Formation in Ferroelectric Negative Capacitance Devices
}

\author{
M. Hoffmann ${ }^{1}$, S. Slesazeck ${ }^{1}$ and T. Mikolajick ${ }^{1,2}$ \\ ${ }^{1}$ NaMLab gGmbH, Noethnitzer Str. 64, Dresden, D-01187, Germany \\ ${ }^{2}$ Chair of Nanoelectronic Materials, TU Dresden, Dresden, D-01069, Germany \\ Email: michael.hoffmann@namlab.com / Phone: (+49) 351-212499016
}

\section{Introduction}

The use of ferroelectric negative capacitance $(\mathrm{NC})$ has been proposed as a promising way to reduce the power dissipation in nanoscale devices [1]. According to single-domain (SD) Landau theory, a hysteresis-free NC state in a ferroelectric might be stabilized in the presence of depolarization fields below a certain critical film thickness $t_{\mathrm{F}, \mathrm{SD}}$. However, it is well-known that depolarization fields will cause the formation of domains in ferroelectrics to reduce the depolarization energy [2], which is rarely considered in the literature on NC [3]. The improvident use of SD Landau theory to model $\mathrm{NC}$ devices seems to be the main reason for the large discrepancy between experimental data and the current theory [4]. Here, we will show by simulation how anti-parallel domain formation can strongly limit the stability of the NC state in a metal-ferroelectric-insulator-metal (MFIM) structure, which is schematically shown in Fig. 1.

\section{Ginzburg-Landau theory}

To correctly model ferroelectric NC devices, the Helmholtz free energy density should be introduced as $h_{F}=\alpha P_{S}^{2}+$ $\beta P_{s}^{4}+k\left(\nabla P_{s}\right)^{2}+\varepsilon_{0} \varepsilon_{b} E_{F}^{2} / 2$, where $\alpha$ and $\beta$ are the Landau coefficients, $P_{s}$ is the spontaneous polarization, $k$ is the domain coupling constant, $E_{\mathrm{F}}$ is the electric field, $\varepsilon_{0}$ is the vacuum permittivity and $\varepsilon_{\mathrm{b}}$ is the relative background permittivity of the ferroelectric. The Ginzburg term $k\left(\nabla P_{s}\right)^{2}$ accounts for the domain wall energy and must be included when considering domain formation. The free energy density of the dielectric is given by $h_{D}=\varepsilon_{0} \varepsilon_{r} E_{D}^{2} / 2$, where $\varepsilon_{\mathrm{r}}$ is the relative permittivity and $E_{\mathrm{D}}$ is the electric field in the dielectric. The total free energy density per unit area of the system is then given by $H=t_{F} h_{F}+t_{D} h_{D}$. The electrostatic boundary conditions are $\varepsilon_{0} \varepsilon_{b} E_{F}+P_{S}=\varepsilon_{0} \varepsilon_{r} E_{D}$ and $V=$ $t_{F} E_{F}+t_{D} E_{D}$, where $V$ is the voltage between the top and bottom electrode.

\section{Stability of the single-domain state}

When $P_{\mathrm{s}}$ in the ferroelectric is homogeneous (i.e. $P_{1}=P_{2}=P_{\mathrm{s}}$ ), the system is stable at $P_{s} \approx 0$ if $\partial^{2} H / \partial P_{s}^{2}>0$. This results in the critical ferroelectric thickness $t_{F, S D}=-\left(1+2 \varepsilon_{0} \varepsilon_{b} \alpha\right) /\left(2 \alpha C_{D}\right)$, where $C_{D}=\varepsilon_{0} \varepsilon_{r} / t_{D}$, below which the $\mathrm{SD}$ state loses its stability with respect to the NC state. Note that this formula slightly differs from the results found in literature, where the influence of the electrostatic self-energy was always neglected $\left(\varepsilon_{b}=0\right)$. Fig. 2 shows the free energy landscapes in the SD case for a) $t_{F}>t_{F, S D}$ and b) $t_{F}<t_{F, S D}$ for $V=0$. In the latter case, the energy minimum of the system is at $P_{\mathrm{s}}=0$, where the NC state in the ferroelectric is stabilized.

\section{Stability of the multi-domain state}

Now we will consider the stability of the anti-parallel multi-domain state, i.e. $P_{1}=-P_{2}=P_{\mathrm{s}}$, which is the more important case, since domain formation will drastically reduce the depolarization energy at the cost of an increase in domain wall energy. The electrostatics are simulated by using an iterative Poisson solver as shown in Fig. 3. For a certain equilibrium domain period $d=d_{\text {eq }}$ (see Fig. 1), the free energy of the total system will have an overall minimum as shown in Fig. 4 a). The simulated spatial distribution of the electrostatic potential $\phi$ in the equilibrium state of Fig. 4 a) is shown in Fig. 5 (periodic boundary conditions were used in x-direction). While $t_{F}<t_{F, S D}$ is true in Fig. 4 a), this does not mean that the NC state is stable. Indeed, our simulations show that there is a different critical thickness $t_{F, M D}<t_{F, S D}$ below which no domains will form. Therefore, $t_{F}<t_{F, M D}<t_{F, S D}$ must be ensured to prevent domain formation and to stabilize $\mathrm{NC}$ as shown in Fig. 4 b), where $P_{\mathrm{s}}=0$ is stable for all domain periods $d$. Furthermore, the dependence of $t_{\mathrm{F}, \mathrm{MD}}$ on the material parameters was investigated. While Fig. 6 shows an inverse square dependence of $t_{\mathrm{F}, \mathrm{MD}}$ on $\alpha$, the dependence on $k / w$ shown in Fig. 7 is linear, where $w$ is the domain wall width. Fig. 8 shows that $t_{\mathrm{F}, \mathrm{MD}}$ is independent of $t_{\mathrm{D}}$, if $t_{\mathrm{D}}$ is larger than the equilibrium domain width $d_{\mathrm{eq}} / 2$. For smaller $t_{\mathrm{D}}$, the ferroelectric polarization will interact with the bottom electrode thus strongly favoring the multi-domain state. Fig. 9 shows that $t_{\mathrm{F}, \mathrm{MD}}$ is inversely proportional to the sum of $\varepsilon_{\mathrm{b}}+\varepsilon_{\mathrm{r}}$. This finally yields the analytic formula for $t_{F, M D}=$ $2.13 k /\left[\alpha^{2} \varepsilon_{0}\left(\varepsilon_{b}+\varepsilon_{r}\right) w\right]$, for larger $t_{\mathrm{D}}$, which was directly derived from the Ginzburg-Landau simulation results. It should be noted that $t_{\mathrm{F}, \mathrm{MD}}$ will give a much more realistic estimation for the critical thickness of the NC state compared to the previous literature. Furthermore, NC amplification might be severely limited when $t_{\mathrm{F}, \mathrm{MD}}<<t_{\mathrm{F}, \mathrm{SD}}$.

[1] S. Salahuddin et al., Nano Lett., vol. 8, p. 405, (2008).

[3] A. Cano et al., Appl. Phys. Lett., vol. 97, p. 133509, (2010).
[2] A. M. Bratkovsky et al., Phys. Rev. B, 63, p. 132103 (2001)

[4] M. Hoffmann et al., EUROSOI-ULIS, Athens, p. 1 (2017). 


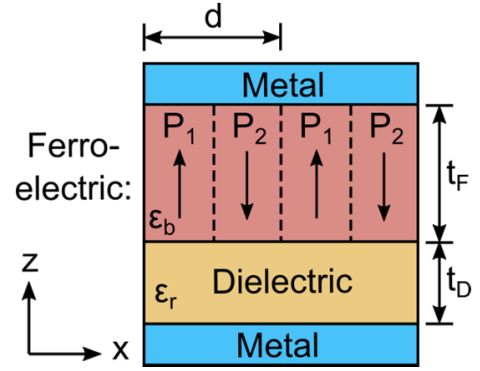

Fig. 1. Schematic of anti-parallel domain formation in an MFIM structure. $d$ is the domain period.

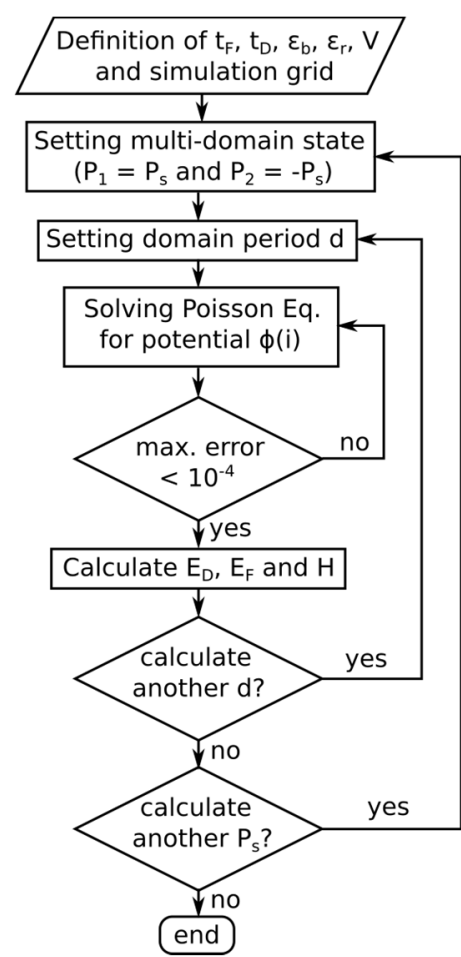

Fig. 3. Flowchart for the multidomain Ginzburg-Landau and electrostatics simulations of the MFIM structure in Fig. 1.

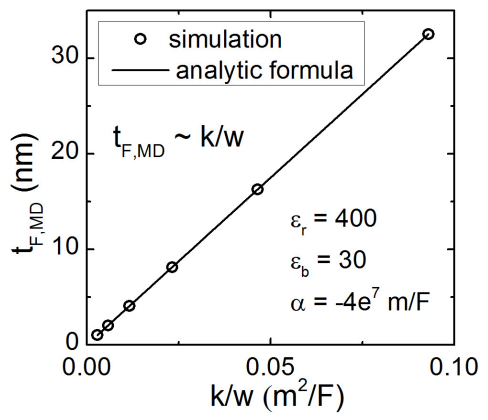

Fig. 7. Comparison of simulation results and the analytic formula for $t_{\mathrm{F}, \mathrm{MD}}$ as a function of $k / w$.
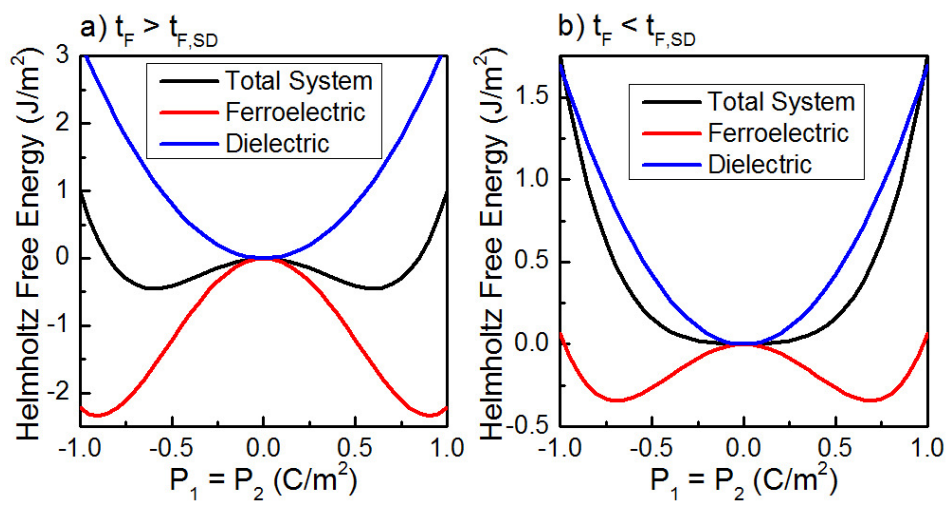

Fig. 2. Free energy densities a) above and b) below the critical ferroelectric thickness $t_{\mathrm{F}, \mathrm{SD}}$ for NC stabilization in the homogeneous single-domain case.

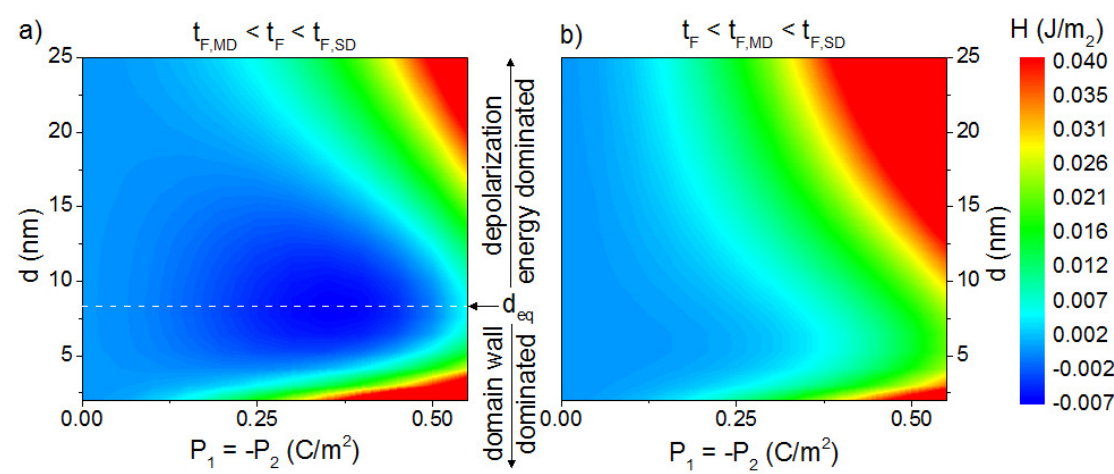

Fig. 4. Total free energy densities a) above and b) below the critical ferroelectric thickness $t_{\mathrm{F}, \mathrm{MD}}$ for $\mathrm{NC}$ stabilization in the multi-domain case.
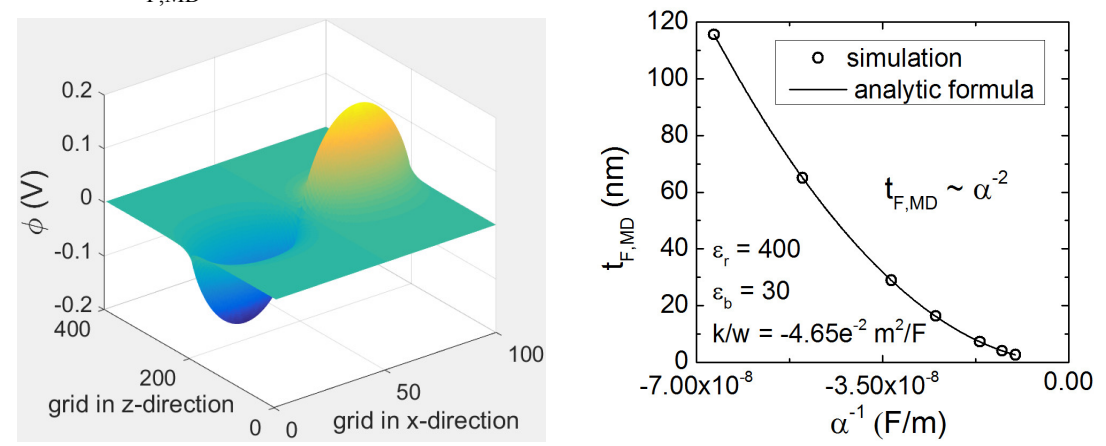

Fig. 5. Distribution of electrostatic Fig. 6. Comparison of simulation potential for one domain period in the minimum energy state in Fig. 4 a).

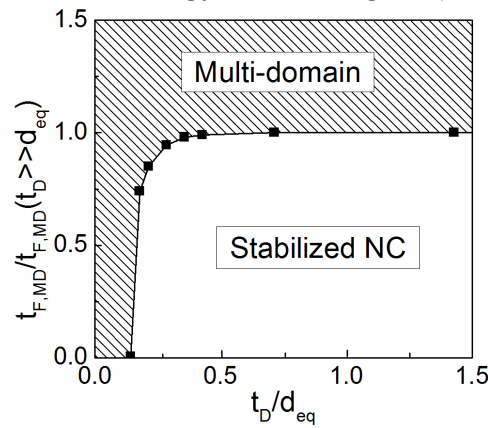

Fig. 8. Stability of the multidomain state as a function of $t_{\mathrm{D}}$. results and the analytic formula for $t_{\mathrm{F}, \mathrm{MD}}$ as a function of $\alpha$.

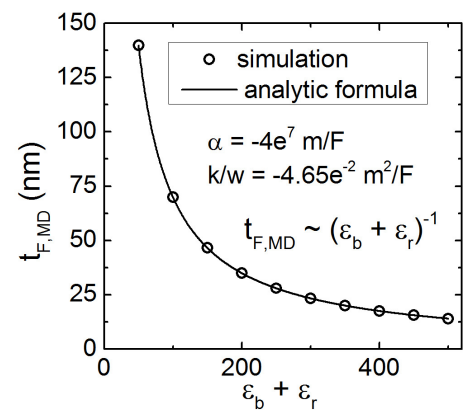

Fig. 9. Comparison of simulation results and the analytic formula for $t_{\mathrm{F}, \mathrm{MD}}$ as a function of $\varepsilon_{\mathrm{b}}+\varepsilon_{\mathrm{r}}$. 